\title{
Upper and lower bounds of the first exit time of Brownian motion from The Minimum and Maximun Parabolic Domains with variable dimension to research the variation of biological species
}

Chao Liu

Qihoo 360 Technology Company, Beijing, China

Wenbin Che

Qihoo 360 Technology Company, Beijing, China

Jingjun Zhang ( $\sim 1910511449 @ q q . c o m$ )

Qihoo 360 Technology Company, Beijing, China https://orcid.org/0000-0002-7025-3789

\section{Research}

Keywords: Brownian motion, Variable dimension, Gordon's inequality

Posted Date: June 23rd, 2020

DOI: https://doi.org/10.21203/rs.3.rs-33770/v1

License: (c) (1) This work is licensed under a Creative Commons Attribution 4.0 International License.

Read Full License 


\title{
Upper and lower bounds of the first exit time of Brownian motion from The Minimum and Maximun Parabolic Domains with variable dimension to research the variation of biological species
}

\begin{abstract}
Consider a Brownian motion with variable dimension starting at an interior point of the minimum or maximum parabolic domains $D_{t}^{\max }$ and $D_{t}^{\min }$ in $R^{d(t)+2}, d(t) \geq 1$ is an increasing integral function as $t \rightarrow \infty, d(t) \rightarrow \infty$, and let $\tau_{D_{t}^{\max }}$ and $\tau_{D_{t}^{\text {min }}}$ denote the first time the Brownian motion exits from $D_{t}^{\max }$ and $D_{t}^{\text {min }}$, respectively. Upper and lower bounds with exact constants for the asymptotics of $\log P\left(\tau_{D_{t}^{\max }}>t\right)$ and $\log P\left(\tau_{D_{t}^{\text {min }}}>t\right)$ are given as $t \rightarrow \infty$, depending on the shape of the domain $D_{t}^{\max }$ and $D_{t}^{\min }$. The methods of proof are based on Gordon's inequality and early works of Li, Lifshits and Shi in the single general parabolic domain case.
\end{abstract}

Keywords: Brownian motion; Variable dimension; Gordon's inequality.

\section{Introduction and main result}

More and more mathematicians have paid attention to the study of the first exit time problem of Brownian motion which has been widely used in mathematics, physics, biology and other fields. Considered that the changes of species which are caused by environmental changes and species migration in ecological chain, the first exit time of Brownian motion is used for research where Brownian motion distribution can be regarded as the change of the number of each species in this paper, and the variable dimension of Brownian motion is considered to species in ecology. It means that species in nature have changed over time. In this paper, we study the first exit time of variable-dimensional Brownian motion in the minimum and maximun parabolic domains to simulate the Early warning function of species change in ecological chain. Throughout the paper, let $\left\{B(s)=B_{1}(s), B_{2}(s), \cdots B_{d(t)}(s)\right\} \in R^{d(t)}, 0 \leq s \leq t$ be a standard $d(t)$-dimensional Brownian motion whose dimensions are changed over time $\mathrm{t}$, where $B_{i}(s), 1 \leq i \leq d(t)$ are independent Brownian motions starting at 0 . Consider the first exit time $\tau_{D_{t}^{\max }}$ and $\tau_{D_{t}^{\text {min }}}$ of $(d(t)+2)$-demensional Brownian motion from the minimum or maximum parabolic domains

$D_{t}^{\min }=\left\{\left(x, y_{1}, y_{2}\right) \in R^{d(t)+2}:\|x\|<\min \left\{\left(y_{1}+1\right)^{1 / p_{1}},\left(y_{2}+1\right)^{1 / p_{2}}\right\}, x \in R^{d(t)}\right\}$, 
and

$D_{t}^{\max }=\left\{\left(x, y_{1}, y_{2}\right) \in R^{d(t)+2}:\|x\|<\max \left\{\left(y_{1}+1\right)^{1 / p_{1}},\left(y_{2}+1\right)^{1 / p_{2}}\right\} x \in R^{d(t)}\right\}$,

where the function $\|x\|:=\left[\sum_{i=1}^{d(t)} x_{i}^{2}\right]^{1 / 2}$ is the Euclidean norm of $x:=\left(x_{1}, \cdots, x_{d(t)}\right) \in$ $R^{d(t)}$. That is the exit time or the stopping time

$$
\begin{aligned}
& \tau_{D_{t}^{\min }}=\inf \left\{s \geq 0 ; B(s) \notin D_{t}^{\min }, 0 \leq s \leq t\right\}, \\
& \tau_{D_{t}^{\max }}=\inf \left\{s \geq 0 ; B(s) \notin D_{t}^{\max }, 0 \leq s \leq t\right\},
\end{aligned}
$$

which plays a key role in the probabilistic solution to the Dirichlet problem.

Before our introduction, let us recall some well-known results of the first exit time. Firstly, Li considered the first exit time of a Brownian motion from an unbounded domain in [4] as follows:

$$
P(f(\|\tilde{B}(s)\|) \leq f(0)+1+W(s), 0 \leq s \leq t), \quad \text { as } t \rightarrow \infty,
$$

where $f(x)$ is convex and symmetric with respect to the set of all orthogonal transformations of $R^{d}$. $\tilde{B}(s), 0 \leq s \leq t$ is a standard d-dimensional Brownian motion, and d is a positive constant. Here and throughout the paper, $W(s)$ is a standard one-dimensional Brownian motion starting at 0 , independent of $\tilde{B}(s)$. Furthermore, he assumed $f(x)$ is a non-decreasing lower semi-continuous convex function on $[0, \infty)$ with $f(0)$ finite as given in [11]. Using the above conditions, very general estimates for the asymptotics of the exit probability were given by Gaussian technique and Slepian's inequality. However, in view of the generality of $f(x)$, the lower and upper estimates were not asymptotically equivalent.

Lifshits and Shi in [5] gave further restrictions and assumed $f(t)=t^{p}$, where $p>1$. They obtained the lower and upper estimates of the following probability

$$
P\left(\|\tilde{B}(s)\|^{p} \leq 1+W(t), 0 \leq s \leq t\right),
$$

and also provided the lower and upper estimates are asymptotically equivalent, thus improving Li's estimates in this case.

On the basis of two results above and Gordon's inequality, Lu [6] considered the exit probabilities in the minimum and maximum parabolic domains, namely

$$
\begin{aligned}
& P\left(\|\tilde{B}(s)\| \leq \min _{j=1,2}\left\{\left(1+W_{j}(s)\right)^{1 / p_{j}}\right\}, 0 \leq s \leq t\right), \\
& P\left(\|\tilde{B}(s)\| \leq \max _{j=1,2}\left\{\left(1+W_{j}(s)\right)^{1 / p_{j}}\right\}, 0 \leq s \leq t\right),
\end{aligned}
$$

where $p_{j}>1, j=1,2$, and $W_{j}, j=1,2$ are standard one-dimensional Brownian motions, not only independent of each other, but also independent of $\left\{B(s) \in R^{d}, s \geq 0\right\}$.

More and more mathematicians have realized the importance of the first exit time. In the last few years, there has been a large number of mathematicians 
to study the first exit time of a Brownian motion from various domains, it was also widely applied in mathematics and physics.

For the sake of clarity, let us recall some well-known results of the first exit time. Firstly, Li considered the first exit time of a Brownian motion from an unbounded domain in [4] as follows:

$$
P(f(\|\tilde{B}(s)\|) \leq f(0)+1+W(s), 0 \leq s \leq t), \quad \text { as } t \rightarrow \infty,
$$

where $f(x)$ is convex and symmetric with respect to the set of all orthogonal transformations of $R^{d}$. $\tilde{B}(s), 0 \leq s \leq t$ is a standard d-dimensional Brownian motion, and $\mathrm{d}$ is a positive constant. Here and throughout the paper, $W(s)$ is a standard one-dimensional Brownian motion starting at 0 , independent of $\tilde{B}(s)$. Furthermore, he assumed $f(x)$ is a non-decreasing lower semi-continuous convex function on $[0, \infty)$ with $f(0)$ finite as given in [11]. Using the above conditions, very general estimates for the asymptotics of the exit probability were given by Gaussian technique and Slepian's inequality. However, in view of the generality of $f(x)$, the lower and upper estimates were not asymptotically equivalent.

Lifshits and Shi in [5] gave further restrictions and assumed $f(t)=t^{p}$, where $p>1$. They obtained the lower and upper estimates of the following probability

$$
P\left(\|\tilde{B}(s)\|^{p} \leq 1+W(t), 0 \leq s \leq t\right),
$$

and also provided the lower and upper estimates are asymptotically equivalent, thus improving Li's estimates in this case.

On the basis of two results above, Lu [6] considered the exit probabilities in the minimum and maximum parabolic domains, namely

$$
\begin{aligned}
& P\left(\|\tilde{B}(s)\| \leq \min _{j=1,2}\left\{\left(1+W_{j}(s)\right)^{1 / p_{j}}\right\}, 0 \leq s \leq t\right), \\
& P\left(\|\tilde{B}(s)\| \leq \max _{j=1,2}\left\{\left(1+W_{j}(s)\right)^{1 / p_{j}}\right\}, 0 \leq s \leq t\right),
\end{aligned}
$$

where $p_{j}>1, j=1,2$, and $W_{j}, j=1,2$ are standard one-dimensional Brownian motions, not only independent of each other, but also independent of $\left\{B(s) \in R^{d}, s \geq 0\right\}$.

It is their works that motivate our study. Up to now, to the best of our knowledge, all the researchers above only consider that the dimension $d$ is a constant. Little is known about the variable dimension. Therefore, in this paper, we mainly consider the exit probabilities of $d(t)$-dimensional Brownian motion, here the dimension changes over time $t$. The dimension is no longer the fixed constant $d$ as previous studies. In practice the problem is more worthy to be studied when the dimension is random. However, this problem is based on the result that $d(t)$ is nonrandom function. Therefore in this paper we consider $d(t)$ is the following case. Throughout the paper, let $\left\{B(s)=B_{1}(s), B_{2}(s), \cdots B_{d(t)}(s)\right\} \in R^{d(t)}, 0 \leq s \leq t$ be a standard $\mathrm{d}(\mathrm{t})$ dimensional Brownian motion whose dimensions are changed over time t, where $B_{i}(s), 1 \leq i \leq d(t)$ are independent Brownian motions starting at 0 . Consider 
the first exit time $\tau_{D_{t}^{\max }}$ and $\tau_{D_{t}^{\text {min }}}$ of $(d(t)+2)$-demensional Brownian motion from the minimum or maximum parabolic domains

$D_{t}^{\min }=\left\{\left(x, y_{1}, y_{2}\right) \in R^{d(t)+2}:\|x\|<\min \left\{\left(y_{1}+1\right)^{1 / p_{1}},\left(y_{2}+1\right)^{1 / p_{2}}\right\}, x \in R^{d(t)}\right\}$,

and

$D_{t}^{\max }=\left\{\left(x, y_{1}, y_{2}\right) \in R^{d(t)+2}:\|x\|<\max \left\{\left(y_{1}+1\right)^{1 / p_{1}},\left(y_{2}+1\right)^{1 / p_{2}}\right\} x \in R^{d(t)}\right\}$,

where the function $\|x\|:=\left[\sum_{i=1}^{d(t)} x_{i}^{2}\right]^{1 / 2}$ is the Euclidean norm of $x:=\left(x_{1}, \cdots, x_{d(t)}\right) \in$ $R^{d(t)}$. That is the exit time or the stopping time

$$
\begin{aligned}
& \tau_{D_{t}^{\min }}=\inf \left\{s \geq 0 ; B(s) \notin D_{t}^{\min }, 0 \leq s \leq t\right\}, \\
& \tau_{D_{t}^{\max }}=\inf \left\{s \geq 0 ; B(s) \notin D_{t}^{\max }, 0 \leq s \leq t\right\},
\end{aligned}
$$

which plays a key role in the probabilistic solution to the Dirichlet problem.

We also consider the exit probabilities in the minimum and maximum parabolic domains, namely

$$
\begin{gathered}
P\left(\tau_{D_{t}^{\text {min }}}>t\right)=P\left(\|B(s)\| \leq \min _{j=1,2}\left\{\left(1+W_{j}(s)\right)^{1 / p_{j}}\right\}, 0 \leq s \leq t\right), \\
P\left(\tau_{D_{t}^{\text {max }}}>t\right)=P\left(\|B(s)\| \leq \max _{j=1,2}\left\{\left(1+W_{j}(s)\right)^{1 / p_{j}}\right\}, 0 \leq s \leq t\right),
\end{gathered}
$$

Note that if $W_{j}(s)<-1, j=1,2$, we assume that probabilities (1.9) and (1.10) are equal to 0 .

In this paper, we provide the asymptotic estimates with exact constants for (1.9) and (1.10). The following simple fact is the key step for our upper estimate of the exit probability (1.10). It is based on a powerful Gaussian technique, Gordon's inequality in [3].

Proposition 1.1. Let $p_{j}>1, j=1,2$, then

$$
\begin{aligned}
& P\left(\|B(s)\| \leq \max _{j=1,2}\left\{\left(1+W_{j}(s)\right)^{1 / p_{j}}\right\}, 0 \leq s \leq t\right) \\
\leq \quad & P\left(\|B(s)\| \leq \max \left\{(1+\sqrt{s} \xi)^{1 / p_{1}},\left(1+W_{2}(s)\right)^{1 / p_{2}}\right\}, 0 \leq s \leq t\right),
\end{aligned}
$$

where $\xi$ is a standard normal random variable and independent of $\{B(s) \in$ $\left.R^{d}, s \geq 0\right\}$ and $W_{2}$.

Gordon's inequality and its variations provide a very useful tool in the theory of Gaussian process and probability in Banach spaces. The simplest form of Gordon's Theorem for centered Gaussian variables $X_{i j}$ and $Y_{i j}$ with indexes $1 \leq i \leq n, 1 \leq j \leq m$ states that if $\mathbf{E}\left(X_{i j}^{2}\right)=\mathbf{E}\left(Y_{i j}^{2}\right)$ for any $i, j, \mathbf{E}\left(X_{i j} X_{i k}\right) \leq$ $\mathbf{E}\left(Y_{i j} Y_{i k}\right)$ for any $i, j, k$, and $\mathbf{E}\left(X_{i j} X_{l k}\right) \geq \mathbf{E}\left(Y_{i j} Y_{l k}\right)$ for any $i \neq l$ and $j, k$, then for all real scalars $\lambda_{i j}$,

$$
P\left(\bigcap_{1 \leq i \leq n} \bigcup_{1 \leq j \leq m}\left\{Y_{i j}>\lambda_{i j}\right\}\right) \leq P\left(\bigcap_{1 \leq i \leq n} \bigcup_{1 \leq j \leq m}\left\{X_{i j}>\lambda_{i j}\right\}\right) .
$$

Using Proposition 1.1, we give the main result of this paper as follows: 
Theorem 1.1. Let $d \geq 1$ and $p_{1}>p_{2}>1$, in the maximum parabolic domain, we have

$$
\begin{aligned}
& \limsup _{t \rightarrow \infty} t^{-\left(p_{2}-1\right) /\left(p_{2}+1\right)}(d(t))^{-\frac{p_{2}}{p_{2}+1}} \log P\left(\tau_{D_{t}^{\max }}>t\right) \\
\leq & -\left(p_{2}+1\right)\left(c_{1} / p_{2}\right)^{p_{2} /\left(p_{2}+1\right)} B_{0, p_{2}}^{1 /\left(p_{2}+1\right)},
\end{aligned}
$$

and

$$
\begin{aligned}
& \liminf _{t \rightarrow \infty} t^{-\left(p_{2}-1\right) /\left(p_{2}+1\right)}(d(t))^{-\frac{2 p_{2}}{p_{2}+1}} \log P\left(\tau_{D_{t}^{\max }}>t\right) \\
\geq & -\left(p_{2}+1\right)\left(c_{2}^{3} / p_{2}\right)^{p_{2} /\left(p_{2}+1\right)} B_{0, p_{2}}^{1 /\left(p_{2}+1\right)}
\end{aligned}
$$

in the minimum parabolic domain, we have

$$
\begin{aligned}
& \limsup _{t \rightarrow \infty} t^{-\left(p_{1}-1\right) /\left(p_{1}+1\right)}(d(t))^{-\frac{p_{1}}{p_{1}+1}} \log P\left(\tau_{D_{t}^{\text {min }}}>t\right) \\
\leq \quad & -\left(p_{1}+1\right)\left(c_{1} / p_{1}\right)^{p_{1} /\left(p_{1}+1\right)} B_{0, p_{1}}^{1 /\left(p_{1}+1\right)} .
\end{aligned}
$$

and

$$
\begin{aligned}
& \liminf _{t \rightarrow \infty} t^{-\left(p_{1}-1\right) /\left(p_{1}+1\right)}(d(t))^{-\frac{2 p_{1}}{p_{1}+1}} \log P\left(\tau_{D_{t}^{\text {min }}}>t\right) \\
\geq & -\left(p_{1}+1\right)\left(c_{2}^{3} / p_{1}\right)^{p_{1} /\left(p_{1}+1\right)} B_{0, p_{1}}^{1 /\left(p_{1}+1\right)} .
\end{aligned}
$$

where

$$
B_{0, p_{j}}=\frac{\pi p_{j}^{p_{j}} \Gamma^{2}\left(\left(p_{j}-1\right) / 2\right)}{8\left(p_{j}-1\right)^{p_{j}-1} \Gamma^{2}\left(p_{j} / 2\right)}, \quad j=1,2,
$$

$\Gamma(\cdot)$ denotes the usual gamma function, and $c_{1}$ and $c_{2}$ are strictly positive constants which are independent of $p$ and $t$.

Remark: Now, we assume that $\widehat{B}(s)=\left\{B_{1}(s), B_{2}(s), \cdots\right\}$ is a infinity dimensional Brownian motion, and the projecting mapping is

$$
\pi_{d(s)}(\widehat{B}(s))=\left\{B_{1}(s), B_{2}(s), \cdots, B_{d(s)}(s), 0, \cdots\right\} .
$$

The generally problem is the estimates of the following probability:

$$
P\left(\sup _{0<s<t}\left\|\pi_{d(s)}(\widehat{B}(s))\right\| \leq x\right) .
$$

On the one hand, it is easy to find that the probability (1.15) is decreasing with $d(s)$, so we have

$$
P\left(\sup _{0<s<t}\left\|\pi_{d(t)}(\widehat{B}(s))\right\| \leq x\right) \leq P\left(\sup _{0<s<t}\left\|\pi_{d(s)}(\widehat{B}(s))\right\| \leq x\right) .
$$

On the other hand, for finite partition $t_{0}=0<t_{1}<t_{2}<\cdots<t_{n}=t$, we can get the upper bound of the the probability (1.15),

$$
\begin{aligned}
P\left(\sup _{0 \leq s \leq t}\left\|\pi_{d(s)}(\widehat{B}(s))\right\| \leq x\right) & =E\left(I_{\cap_{i=1}^{n}\left\{\sup _{t_{i-1}<s<t_{i}}\left\|\pi_{d(s)}(\widehat{B}(s))\right\| \leq x\right\}}\right) \\
& \leq E\left(I_{\cap_{i=1}^{n}\left\{\sup _{t_{i-1}<s<t_{i}}\left\|\pi_{d\left(t_{i-1}\right)}(\widehat{B}(s))\right\| \leq x\right\}}\right) .
\end{aligned}
$$


Using conditional expectation, we have

$$
\begin{aligned}
& E\left(I_{\cap_{i=1}^{n}\left\{\sup _{t_{i-1}<s<t_{i}}\left\|\pi_{d\left(t_{i-1}\right)}(\widehat{B}(s))\right\| \leq x\right\}}\right) \\
= & E\left(E\left(I_{\cap_{i=1}^{n}\left\{\sup _{t_{i-1}<s<t_{i}}\left\|\pi_{d\left(t_{i-1}\right)}(\widehat{B}(s))\right\| \leq x\right\}} \mid \mathcal{F}_{t_{n-1}}\right)\right) \\
= & E\left(I_{\cap_{i=1}^{n-1}\left\{\sup _{t_{i-1}<s<t_{i}}\left\|\pi_{d\left(t_{i-1}\right)}(\widehat{B}(s))\right\| \leq x\right\}} E\left(I_{\left\{\sup _{t_{n-1}<s<t_{n}}\left\|\pi_{d\left(t_{n-1}\right)}(\widehat{B}(s))\right\| \leq x\right\}} \mid \mathcal{F}_{t_{n-1}}\right)\right),
\end{aligned}
$$

where $\mathcal{F}_{t}=\sigma(\widehat{B}(s), 0 \leq s \leq t)$. By Anderson's inequality for Gaussian measure and scaling property of Brownian motion,

$$
\begin{aligned}
& E\left(I_{\left\{\sup _{t_{n-1}<s<t_{n}}\left\|\pi_{d\left(t_{n-1}\right)}(\widehat{B}(s))\right\| \leq x\right\}} \mid \mathcal{F}_{t_{n-1}}\right) \\
= & P\left(\sup _{t_{n-1}<s<t_{n}}\left\|\pi_{d\left(t_{n-1}\right)}\left(\widehat{B}(s)-\widehat{B}\left(t_{n-1}\right)+\widehat{B}\left(t_{n-1}\right)\right)\right\| \leq x \mid \mathcal{F}_{t_{n-1}}\right) \\
\leq & P\left(\sup _{t_{n-1}<s<t_{n}}\left\|\pi_{d\left(t_{n-1}\right)}\left(\widehat{B}(s)-\widehat{B}\left(t_{n-1}\right)\right)\right\| \leq x\right) \\
= & P\left(\sup _{0<s<t_{n}-t_{n-1}}\left\|\pi_{d\left(t_{n-1}\right)}(\widehat{B}(s))\right\| \leq x\right) .
\end{aligned}
$$

Then, by induction, we have

$$
P\left(\sup _{0<s<t}\left\|\pi_{d(s)}(\widehat{B}(s))\right\| \leq x\right) \leq \prod_{i=1}^{n} P\left(\sup _{0<s<t_{i}-t_{i-1}}\left\|\pi_{d\left(t_{i-1}\right)}(\widehat{B}(s))\right\| \leq x\right) .
$$

Thus combining (1.16) and (1.17), for calculating (1.15) we only consider the simple case

$$
P\left(\sup _{0<s<t}\left\|\pi_{d(t)}(\widehat{B}(s))\right\| \leq x\right) .
$$

Thus, in this paper we just consider the dimension $d(t)$ instead of dimension $d(s)$.

The rest of the paper is organized as follows. In Section 2, we present several estimates for the Brownian motion and the Bessel process of the exit probability for moving boundaries. They are necessary for the proof of Theorem 1.1. In Section 3, we give the proofs of Proposition 1.1 and the upper estimates in (1.13) and (1.11). The proofs of the lower estimates in (1.12) and (1.14) are presented in Section 4.

\section{Exit probabilities with moving boundary}

To prove Theorem 1.1, in this section, we need the following results due to Shao and Wang [9] for the small ball probabilities in Gaussian field, the result 
for the first exit time of Brownian motion from a unbounded convex domain in $\mathrm{Li}$ [4], and the result for the first exit time of Brownian motion from a parabolic domain in Lifshits and Shi [5].

If the dimension is a constant $d$, we consider the probability

$$
P\left(\sup _{0 \leq s \leq t}\|\tilde{B}(s)\| \leq x\right) .
$$

From $\mathrm{Li}[4]$, it is easy to see, for any $x>0$,

$$
K^{-1} \exp \left\{-\frac{j_{v}^{2}}{2 x^{2}}\right\} \leq P\left(\sup _{0 \leq s \leq 1}|\tilde{B}(s)| \leq x\right) \leq K \exp \left\{-\frac{j_{v}^{2}}{2 x^{2}}\right\}
$$

where $K$ is a various positive constant, and $j_{v}$ is the smallest positive zero of the Bessel function $J_{v}, v=(d-2) / 2$.

However, up to now, we don't know any result for the dimension $d(t)$. So, in this section, we give two lemmas for getting upper and lower bounds of the probability

$$
P\left(\sup _{0 \leq s \leq t}\|B(s)\| \leq x\right)
$$

Lemma 2.1. Let $d \geq 1,\left\{Z(t) ; t \in R^{d}\right\}$ be a functional Levy Brownian fields of order $\alpha, 0<\alpha<2$, i.e.

$$
E(Z(s)-Z(t))^{2}=\|s-t\|^{2 \alpha},
$$

for all $s, t \geq 0$. Then there exists $0<c_{1}<\infty$ depending only on $\alpha$ and $d$ such that

$$
P\left\{\sup _{0 \leq t \leq 1}|Z(t)| \leq x\right\} \leq \exp \left(-\frac{c_{1}}{x^{d / \alpha}}\right)
$$

for any $0<x<1$

The argument of Lemma 2.1 was given in Shao and Wang [9]. And they also gave a lower bound of small ball probability of Gaussian fields.

Lemma 2.2. Let $X=\left\{X(t) ; t \in[0,1]^{d}\right\}$ be a Gaussian field with mean zero. Assume that there exits a non-decreasing function $\sigma(x)$ on $[0,1]$ such that

$$
E|X(t)-X(s)|^{2} \leq \sigma^{2}(\|t-s\|),
$$

for every $s, t \in[0,1]^{d}$.

Suppose that $\alpha>0, \sigma(x) / x^{\alpha}$ is non-decreasing on $[0,1]$ for some $\alpha>0$ and that

$$
\sigma(k h) \leq k^{2} \sigma(h)
$$

for every $0<h \leq 1$ and integer $k$ with $0 \leq k \leq 1 / h$. Then there exists a positive constant $c_{2}=c_{2}(\alpha, d)$, for any $0<x<1$

$$
P\left\{\sup _{0 \leq t \leq 1}|X(t)| \leq c_{2} \sigma(x)\right\} \geq \exp \left(-\frac{c_{2}}{x^{d / \alpha}}\right) .
$$


In fact, Shao in [8] obtained estimate of the probability $P\left(\sup _{0 \leq s \leq t}\|B(s)\| \leq\right.$ $x)$ with exact constants. However, we don't care about the precise coefficients in this paper. So we just use the above Lemmas.By Lemma 2.1 and Lemma 2.2, we get the following Propositions.

Proposition 2.1. $\{B(s), 0 \leq s \leq t\}$ be a $d(t)$-dimensional Brownian motion, $d(t) \geq 1$. Then there exist constants $0<c_{1}<c_{2}<\infty$, such that

$$
\exp \left(-\frac{c_{2}^{3} t d^{2}(t)}{x^{2}}\right) \leq P\left(\sup _{0 \leq s \leq t}\|B(s)\| \leq x\right) \leq \exp \left(-\frac{c_{1} t d(t)}{x^{2}}\right) .
$$

Proof: First, we use the independence between $B_{i}$

$$
\begin{aligned}
P\left(\sup _{0 \leq s \leq t}\|B(s)\| \leq x\right) & \leq P\left(\sup _{0 \leq s \leq t}\left|B_{i}(s)\right| \leq x, i=1,2, \ldots d(t)\right) \\
& =P\left(\sup _{0 \leq s \leq 1}\left|B_{1}(s)\right| \leq \frac{x}{\sqrt{t}}\right)^{d(t)} .
\end{aligned}
$$

By Lemma 2.1, let $d=1, \alpha=1 / 2$, and $Z(t)$ defined as $B_{1}(t)$, then (2.1) can be written as

$$
P\left(\sup _{0 \leq s \leq 1}\left|B_{1}(s)\right| \leq \frac{x}{\sqrt{t}}\right) \leq \exp \left(-\frac{c_{1} t}{x^{2}}\right) .
$$

Plugging (2.5) into (2.4), we have

$$
P\left(\sup _{0 \leq s \leq t}\|B(s)\| \leq x\right) \leq \exp \left(-\frac{c_{1} t d(t)}{x^{2}}\right) .
$$

Next, we consider the lower bound of the probability.

$$
\begin{aligned}
& P\left(\sup _{0 \leq s \leq t}\|B(s)\| \leq x\right) \\
\geq & P\left(\sup _{0 \leq s \leq t}\left|B_{i}(s)\right| \leq \frac{x}{\sqrt{d(t)}}, i=1,2, \ldots, d(t)\right) \\
= & P\left(\sup _{0 \leq s \leq 1}\left|B_{i}(s)\right| \leq \frac{x}{\sqrt{t d(t)}}, i=1,2, \ldots, d(t)\right) .
\end{aligned}
$$

By Lemma 2.2, let $\alpha=\frac{1}{2}, d=1, \sigma(x)=\sqrt{x}$, then the inequality (2.7) can be written as

$$
P\left(\sup _{0 \leq s \leq 1}\left|B_{i}(s)\right| \leq \frac{x}{\sqrt{t d(t)}}\right) \geq \exp \left(-\frac{c_{2}^{3} t d(t)}{x^{2}}\right) .
$$

Plugging (2.8) into (2.7)

$$
P\left(\sup _{0 \leq s \leq t}\|B(s)\| \leq x\right) \geq \exp \left(-\frac{c_{2}^{3} t d^{2}(t)}{x^{2}}\right) .
$$

Combining (2.6) and (2.9), we have the inequality (2.3). 
The inequality (2.3) is very important for the proof of the Theorem 1.1.

Similarly to Theorem 2.2 in Li [4], using Proposition 2.1 we give a result for variable dimension $d(t)$.

Proposition 2.2. Let $g(t)$ be a continuous strictly positive function such that $g^{\prime \prime}(t) \leq 0$ is continuous and $g^{\prime}(t) \geq 0$. Then

$$
\begin{aligned}
& P(\|B(s)\| \leq g(s), 0 \leq s \leq t) \\
& \geq \exp \left\{\frac{d(t)}{2} \frac{g(t)}{g(0)}-\frac{1}{2} \int_{0}^{t}\left(g^{\prime}(s)\right)^{2} d s-c_{2}^{3} d^{2}(t) \int_{0}^{t} \frac{1}{g^{2}(s) d s}\right\},
\end{aligned}
$$

here $c_{2}$ is a sufficiently large constant.

In particular, under the additional condition $\sqrt{t} g^{\prime}(t) \rightarrow 0$ as $t \rightarrow \infty$,

$$
\log P(\|B(s)\| \leq g(s), 0 \leq s \leq t) \geq-(1+\delta) c_{2}^{3} d^{2}(t) \int_{0}^{t} g^{-2}(s) d s .
$$

for and $\delta>0$ and $t$ large.

The proof of the Proposition 2.2 is based on proof Theorem 2.2 in [4], we only replaced (2.18) in $\mathrm{Li}[4]$ by the following inequality, using Brownian scaling property and (2.3), we have

$$
\begin{aligned}
& P(\|\Xi(s)\| \leq g(s), 0 \leq s \leq t) \\
& =P\left(\left\|g(s) \int_{0}^{t} G(s) d B(s)\right\| \leq g(s), 0 \leq s \leq t\right) \\
& =P\left(\|B(s)\| \leq 1,0 \leq s \leq \int_{0}^{t} \frac{d s}{g^{2}(s)}\right) \\
& =P\left(\sup _{0 \leq s \leq 1}|B(s)| \leq\left(\int_{0}^{t} \frac{d s}{g^{2}(s)}\right)^{-1 / 2}\right) \\
& \geq \exp \left\{-c_{2}^{3} d^{2}(t) \int_{0}^{t} \frac{d s}{g^{2}(s)}\right\} .
\end{aligned}
$$

The rest proof is similar to the proof of Theorem 2.2 in Li [4].

Lemma 2.3. Let $g(s)$ be an absolutely continuous function, with $g(0)<0$ and

$$
\int_{0}^{t} \dot{g}^{2}(s) d s<\infty \quad \text { for } \quad t<\infty
$$

For any $p>1$

$$
P(W(s) \geq g(s), 0 \leq s \leq t) \geq\left(\Phi_{0}\left(\frac{|g(0)|}{\sqrt{t}}\right)\right)^{p /(p-1)} \cdot \exp \left\{-\frac{p}{2} \int_{0}^{t} \dot{g}^{2}(s) d s\right\} .
$$

Here

$$
\Phi_{0}(x)=(2 / \pi)^{1 / 2} \int_{0}^{x} \exp \left\{-y^{2} / 2\right\} d y=2 \Phi(x)-1 \quad(x \geq 0),
$$

where $\dot{g}$ denotes the Radon-Nikodym derivative of $g$ and $\Phi(x)$ is the distribution function of a standard normal random variable. 
Lemma 2.3 is given in [7] and follows from well-known theorems concerning the equivalence of measures of Gaussian processes. Note that

$$
\Phi_{0}(|g(0)| / \sqrt{t}) \sim|g(0)|(2 / \pi t)^{1 / 2}, \quad t \rightarrow \infty .
$$

Before giving more lemmas, let us introduce some notation. Here and in the following,

$$
B_{\theta, p}:=\frac{1}{2} \inf _{f \in A_{\theta, p}^{\uparrow}} \int_{0}^{1} \dot{f}^{2}(s) d s,
$$

where $A_{\theta, p}^{\uparrow}$ is the set of all non-decreasing functions on the set $A_{\theta, p}$ defined by

$A_{\theta, p}:=\left\{f:[0,1] \rightarrow R_{+}, f(0)=0, f\right.$ absolutely continuous, $\left.\int_{\theta}^{1} f^{-2 / p}(s) d s \leq 1\right\}$.

Using the above notation and applying the classical Schilder large deviation theorem, Lishifts and Shi provided a useful upper estimate in [5].

Lemma 2.4. For $p>1$ and $\theta>0$, we have

$\limsup _{\lambda \rightarrow \infty} \lambda^{-p /(p+1)} \log \boldsymbol{E}\left\{\exp \left(-\lambda \int_{\theta}^{1} Q^{-2 / p}(s) d s\right)\right\} \leq-(p+1) p^{-p /(p+1)} B_{\theta, p}^{1 /(p+1)}$,

where $Q(t):=\sup _{0 \leq s \leq t} W(s)$.

Using Lemma 2.4, Song [10] obtained the upper and lower estimates of the exit probability (1.6) of brownian motion from a parabolic domain with variable dimension $d(t)$, as follows:

Lemma 2.5. Let $d(t) \geq 1, p>1$, we have,

$$
\begin{aligned}
& \limsup _{t \rightarrow \infty} t^{-\frac{p-1}{p+1}}(d(t))^{-\frac{p}{p+1}} \log P\left(\|B(s)\|^{p} \leq 1+W(s), 0 \leq s \leq t\right) \\
\leq & -(p+1)\left(\frac{c_{1}^{p} \pi}{8(p-1)^{p-1}} \cdot \frac{\Gamma^{2}((p-1) / 2)}{\Gamma^{2}(p / 2)}\right)^{\frac{1}{p+1}}
\end{aligned}
$$

and

$$
\begin{aligned}
& \liminf _{t \rightarrow \infty} t^{-\frac{p-1}{p+1}}(d(t))^{-\frac{2 p}{p+1}} \log P\left(\|B(s)\|^{p} \leq 1+W(s), 0 \leq s \leq t\right) \\
\geq & -(p+1)\left(\frac{c_{2}^{3 p} \pi}{8(p-1)^{p-1}} \cdot \frac{\Gamma^{2}((p-1) / 2)}{\Gamma^{2}(p / 2)}\right)^{\frac{1}{p+1}}
\end{aligned}
$$

where $\Gamma(\cdot)$ denotes the usual gamma function, and $c_{1}$ and $c_{2}$ are strictly positive constants which are independent of $p$ and $t$.

Using Lemma 2.1-2.5 in the next two sections, we give the proof of Theorem 1.1 . 


\section{Upper estimates}

Our upper bounds argument modifies the one appeared in Lifshits and Shi [5]. We need to pay special attention to the variable dimension $d(t)$ and inequality (2.3). It is easy to obtain the upper estimate of (1.13). Note that

$$
\begin{aligned}
& P\left(\|B(s)\| \leq \min _{j=1,2}\left\{\left(1+W_{j}(s)\right)^{1 / p_{j}}\right\}, 0 \leq s \leq t\right) \\
= & P\left(\bigcap_{j=1,2}\left\{\|B(s)\|^{p_{j}} \leq 1+W_{j}(s), 0 \leq s \leq t\right\}\right) .
\end{aligned}
$$

Thus we only need to provide the upper estimate of the probability on the right-hand side of (3.1). It is easy to see that

$$
\begin{aligned}
& P\left(\bigcap_{j=1,2}\left\{\|B(s)\|^{p_{j}} \leq 1+W_{j}(s), 0 \leq s \leq t\right\}\right) \\
\leq & \min _{j=1,2}\left\{P\left(\|B(s)\|^{p_{j}} \leq 1+W_{j}(s), 0 \leq s \leq t\right)\right\} .
\end{aligned}
$$

For the probability on the right-hand side of (3.2), we use Lemma 2.5 to find the upper bounds of two probabilities, and compare them to get the upper estimate in (1.13) as follows:

$$
\begin{aligned}
& \limsup _{t \rightarrow \infty} t^{-\left(p_{1}-1\right) /\left(p_{1}+1\right)}(d(t))^{-\frac{p_{1}}{p_{1}+1}} \log P\left(\|B(s)\| \leq \min _{j=1,2}\left\{\left(1+W_{j}(s)\right)^{1 / p_{j}}\right\}, 0 \leq s \leq t\right) \\
\leq & -\left(p_{1}+1\right)\left(\frac{c_{1}^{p_{1}} \pi}{8\left(p_{1}-1\right)^{p_{1}-1}} \cdot \frac{\Gamma^{2}\left(\left(p_{1}-1\right) / 2\right)}{\Gamma^{2}\left(p_{1} / 2\right)}\right)^{p_{1} /\left(p_{1}+1\right)}
\end{aligned}
$$

Before giving the upper estimate in (1.11), we need to prove Proposition 1.1. We have

$$
\begin{aligned}
& P\left(\|B(s)\| \leq \max _{j=1,2}\left\{\left(1+W_{j}(s)\right)^{1 / p_{j}}\right\}, 0 \leq s \leq t\right) \\
= & P\left(\bigcup_{j=1,2}\left\{W_{j}(s)-\|B(s)\|^{p_{j}} \geq-1\right\}, 0 \leq s \leq t\right) \\
\leq & P\left(\left\{\sqrt{s} \xi-\|B(s)\|^{p_{1}} \geq-1\right\} \bigcup\left\{W_{2}(s)-\|B(s)\|^{p_{2}} \geq-1\right\}, 0 \leq s \leq t\right) \\
= & P\left(\|B(s)\| \leq \max \left\{(1+\sqrt{s} \xi)^{1 / p_{1}},\left(1+W_{2}(s)\right)^{1 / p_{2}}\right\}, 0 \leq s \leq t\right),
\end{aligned}
$$

where the inequality follows from Gordon's Theorem [3] by conditioning on $B(s), 0 \leq s \leq t$. To justify it, we simply note that $Y_{i j}=W_{j}\left(s_{i}\right), X_{i 1}=$ $\sqrt{s_{i}} \xi, X_{i 2}=W_{2}\left(s_{i}\right), s_{i} \in[0, t]$ is a rational number, $j=1,2, i \in N$. It is easy to verify that $\mathbf{E}\left(X_{i j}^{2}\right)=\mathbf{E}\left(Y_{i j}^{2}\right)=s_{i}$, for any $i, j$, and $\mathbf{E}\left(Y_{i j} Y_{i k}\right)=\mathbf{E}\left(X_{i j} X_{i k}\right)=$ $s_{i} \delta_{j k}$, for any $i, j, k$,

$$
\mathbf{E}\left(Y_{i j} Y_{l k}\right)=\min \left\{s_{i}, s_{l}\right\} \delta_{j k} \leq \mathbf{E}\left(X_{i j} X_{l k}\right) \quad \text { for any } i \neq l \text {, and } j, k,
$$

where $j=k, \delta_{j k}=1$ and $j \neq k, \delta_{j k}=0$. Thus, Proposition 1.1 follows from Gordon's inequality. Note that in most papers and books Gordon's inequality 
is proved and used for mean-zero Gaussian random vectors. Here we, in fact, use a form that the same mean depends on the index parameter. The standard proof can be modified to cover this case.

Next, using (3.4), we give the proof of the upper bound estimate in (1.11). Our argument is a modification of the one from [5]. To obtain a rigorous upper bound, let $0=t_{0}<t_{1}<t_{2}<\ldots<t_{M} \leq t$ and observe that

$$
\begin{aligned}
& P\left(\|B(s)\| \leq \max \left\{(1+\sqrt{s} \xi)^{1 / p_{1}},\left(1+W_{2}(s)\right)^{1 / p_{2}}\right\}, 0 \leq s \leq t\right) \quad \\
\leq & P\left(\sup _{t_{i-1} \leq s \leq t_{i}}\|B(s)\| \leq \max \left\{\left(1+S\left(t_{i}\right)\right)^{1 / p_{1}},\left(1+S_{2}\left(t_{i}\right)\right)^{1 / p_{2}}\right\}, i \leq M\right),
\end{aligned}
$$

where $S(t):=\sup _{0 \leq s \leq t} \sqrt{s} \xi$ and $S_{2}(t):=\sup _{0 \leq s \leq t} W_{2}(s)$. Since $B(s) \in R^{d}$ has independent increments, we have for $a_{i}>0, i \leq \bar{M}$,

$$
\begin{aligned}
& P\left\{\sup _{t_{i-1} \leq s \leq t_{i}}\|B(s)\| \leq a_{i}, 1 \leq i \leq M\right\} \\
= & P\left(\bigcap_{1 \leq i \leq M-1} A_{i},\|B(s)\| \leq a_{M}, s \in\left[t_{M-1}, t_{M}\right]\right) \\
= & E\left(1_{\left\{\bigcap_{i=1}^{M-1} A_{i}\right\}} P\left(\left\|B(s)-B\left(t_{M-1}\right)+x\right\| \leq a_{M}, s \in\left[t_{M-1}, t_{M}\right] \mid B\left(t_{M-1}\right)=x\right)\right),
\end{aligned}
$$

where

$$
A_{i}=\left\{\|B(s)\| \leq a_{i}, t_{i-1} \leq s \leq t_{i}\right\}, \quad 1 \leq i \leq M .
$$

By Anderson's inequality and the fact that $B$ has stationary increments, we have

$$
\begin{aligned}
& P\left(\left\|B(s)-B\left(t_{M-1}\right)+x\right\| \leq a_{M}, t_{M-1} \leq s \leq t_{M}\right) \\
\leq & P\left(\left\|B(s)-B\left(t_{M-1}\right)\right\| \leq a_{M}, t_{M-1} \leq s \leq t_{M}\right) \\
= & P\left(\|B(s)\| \leq a_{M}, 0 \leq s \leq t_{M}-t_{M-1}\right) .
\end{aligned}
$$

Plugging (3.7) into (3.6), by induction, we have

$$
P\left\{\sup _{t_{i-1} \leq s \leq t_{i}}\|B(s)\| \leq a_{i}, 1 \leq i \leq M\right\} \leq \prod_{1 \leq i \leq M} P\left\{\sup _{0 \leq s \leq t_{i}-t_{i-1}}\|B(s)\| \leq a_{i}\right\} .
$$

By Proposition 2.1, we know that

$$
P\left\{\sup _{0 \leq s \leq t}\|B(s)\| \leq x\right\} \leq \exp \left(-\frac{c_{1} d(t)}{x^{2}} t\right), \quad x \rightarrow 0^{+},
$$

Using (3.8) and (3.9), we have

$$
P\left\{\sup _{t_{i-1} \leq s \leq t_{i}}\|B(s)\| \leq a_{i}, 1 \leq i \leq M\right\} \leq \exp \left(-\frac{c(\varepsilon)}{1-\varepsilon} \sum_{1 \leq i \leq M} \frac{t_{i}-t_{i-1}}{a_{i}^{2}}\right),
$$


where $c(\varepsilon)=(1-\varepsilon) c_{1} d(t)$. By conditioning on the Brownian motion $W_{2}$ and the standard normal variable $\xi$, and using (3.5) and (3.10), we have

$$
\begin{gathered}
P\left(\|B(s)\| \leq \max \left\{(1+\sqrt{s} \xi)^{1 / p_{1}},\left(1+W_{2}(s)\right)^{1 / p_{2}}\right\}, 0 \leq s \leq t\right) \\
\leq \mathbf{E}\left\{\exp \left(-\frac{c(\varepsilon)}{1-\varepsilon} \sum_{1 \leq i \leq M} \frac{t_{i}-t_{i-1}}{\max \left\{\left(1+S\left(t_{i}\right)\right)^{2 / p_{1}},\left(1+S_{2}\left(t_{i}\right)\right)^{2 / p_{2}}\right\}}\right)\right\}
\end{gathered}
$$

We split the expectation in (3.11) into two parts

$$
\begin{aligned}
& I=\mathbf{E}\left\{\exp \left(-\frac{c(\varepsilon)}{1-\varepsilon} \sum_{1 \leq i \leq M} \frac{t_{i}-t_{i-1}}{\max \left\{\left(1+S\left(t_{i}\right)\right)^{2 / p_{1}},\left(1+S_{2}\left(t_{i}\right)\right)^{2 / p_{2}}\right\}}\right) 1_{\{\xi \leq 0\}}\right\}, \\
& I I=\mathbf{E}\left\{\exp \left(-\frac{c(\varepsilon)}{1-\varepsilon} \sum_{1 \leq i \leq M} \frac{t_{i}-t_{i-1}}{\max \left\{\left(1+S\left(t_{i}\right)\right)^{2 / p_{1}},\left(1+S_{2}\left(t_{i}\right)\right)^{2 / p_{2}}\right\}}\right) 1_{\{\xi>0\}}\right\} .
\end{aligned}
$$

In view of $S_{2}(t) \geq 0$ for $t \geq 0$, in the case of $\xi \leq 0$, we have $\left(1+\sup _{0 \leq s \leq t_{i}} \sqrt{s} \xi\right)^{2 / p_{1}} \leq$ $\left(1+S_{2}\left(t_{i}\right)\right)^{2 / p_{2}}$ for $1 \leq i \leq M$. Using the independence between $W_{2}$ and $\xi$, it is easy to see

$$
\begin{aligned}
I & =\mathbf{E}\left\{\exp \left(-\frac{c(\varepsilon)}{1-\varepsilon} \sum_{1 \leq i \leq M} \frac{t_{i}-t_{i-1}}{\left(1+S_{2}\left(t_{i}\right)\right)^{2 / p_{2}}}\right) 1_{\{\xi \leq 0\}}\right\} \\
& =\frac{1}{2} \mathbf{E}\left\{\exp \left(-\frac{c(\varepsilon)}{1-\varepsilon} \sum_{1 \leq i \leq M} \frac{t_{i}-t_{i-1}}{\left(1+S_{2}\left(t_{i}\right)\right)^{2 / p_{2}}}\right)\right\} .
\end{aligned}
$$

On the other hand, for $I I$, let $\tau_{i}=t_{i} / t=(1-\varepsilon)^{M-i}, 1 \leq i \leq M$. In the case of $\xi>0$, by the scaling property of $W_{2}$ and the monotonicity of $S_{2}(t)$, we have

$$
\begin{aligned}
& \sum_{1 \leq i \leq M} \frac{t_{i}-t_{i-1}}{\max \left\{\left(1+\sup _{0 \leq s \leq t_{i}} \sqrt{s} \xi\right)^{2 / p_{1}},\left(1+S_{2}\left(t_{i}\right)\right)^{2 / p_{2}}\right\}} \\
= & t \sum_{1 \leq i \leq M} \frac{\tau_{i}-\tau_{i-1}}{\max \left\{\left(1+\sqrt{t} \sqrt{\tau_{i}} \xi\right)^{2 / p_{1}},\left(1+\sqrt{t} S_{2}\left(\tau_{i}\right)\right)^{2 / p_{2}}\right\}} \\
\geq & t \sum_{1 \leq i \leq M-1} \frac{(1-\varepsilon)\left(\tau_{i+1}-\tau_{i}\right)}{\max \left\{\left(1+\sqrt{t} \sqrt{\tau_{i}} \xi\right)^{2 / p_{1}},\left(1+\sqrt{t} S_{2}\left(\tau_{i}\right)\right)^{2 / p_{2}}\right\}} \\
\geq & (1-\varepsilon) t \sum_{1 \leq i \leq M-1} \int_{\tau_{i}}^{\tau_{i+1}} \frac{d \tau}{\max \left\{(1+\sqrt{t} \sqrt{\tau} \xi)^{2 / p_{1}},\left(1+\sqrt{t} S_{2}(\tau)\right)^{2 / p_{2}}\right\}} \\
\geq & (1-\varepsilon) t \int_{\tau_{1}}^{1} \frac{d \tau}{\max \left\{(1+\sqrt{t} \xi)^{2 / p_{1}},\left(1+\sqrt{t} S_{2}(\tau)\right)^{2 / p_{2}}\right\}} .
\end{aligned}
$$


Plugging (3.13) into $I I$ yields

$$
I I \leq \mathbf{E}\left\{\exp \left(-c(\varepsilon) t \int_{\tau_{1}}^{1} \frac{d \tau}{\max \left\{(1+\sqrt{t} \xi)^{2 / p_{1}},\left(1+\sqrt{t} S_{2}(\tau)\right)^{2 / p_{2}}\right\}}\right) 1_{\{\xi>0\}}\right\} .
$$

Similarly, from (3.12) we also obtain

$$
I \leq \frac{1}{2} \mathbf{E}\left\{\exp \left(-c(\varepsilon) t \int_{\tau_{1}}^{1} \frac{d \tau}{\left(1+\sqrt{t} S_{2}(\tau)\right)^{2 / p_{2}}}\right)\right\} .
$$

Next, we deal with $I I$. We split the expectation in (3.14) into two parts,

$$
\begin{aligned}
& I I I=\mathbf{E}\left\{\exp \left(-c(\varepsilon) t \int_{\tau_{1}}^{1} \frac{d \tau}{\max \left\{(1+\sqrt{t} \xi)^{2 / p_{1}},\left(1+\sqrt{t} S_{2}(\tau)\right)^{2 / p_{2}}\right\}}\right) 1_{\{\xi>0, Q\}}\right\}, \\
& I V=\mathbf{E}\left\{\exp \left(-c(\varepsilon) t \int_{\tau_{1}}^{1} \frac{d \tau}{\max \left\{(1+\sqrt{t} \xi)^{2 / p_{1}},\left(1+\sqrt{t} S_{2}(\tau)\right)^{2 / p_{2}}\right\}}\right) 1_{\left\{\xi>0, Q^{c}\right\}}\right\},
\end{aligned}
$$

where $Q=\left\{(1+\sqrt{t} \xi)^{2 / p_{1}} \leq\left(1+\sqrt{t} S_{2}\left(2 \tau_{1}\right)\right)^{2 / p_{2}}\right\}$. In the case of $\xi>0$ and $Q$, in view of the monotonicity of $S_{2}(t)$, for the integral in $I I I$, we have for M large,

$$
\int_{\tau_{1}}^{1} \frac{d \tau}{\max \left\{(1+\sqrt{t} \xi)^{2 / p_{1}},\left(1+\sqrt{t} S_{2}(\tau)\right)^{2 / p_{2}}\right\}} \geq \int_{2 \tau_{1}}^{1} \frac{d \tau}{\left(1+\sqrt{t} S_{2}(\tau)\right)^{2 / p_{2}}} .
$$

Using the independence between $\xi$ and $S_{2}(t)$, and plugging (3.16) into $I I I$ yields

$$
\begin{aligned}
I I I & \leq \mathbf{E}\left\{\exp \left(-c(\varepsilon) t \int_{2 \tau_{1}}^{1} \frac{d \tau}{\left(1+\sqrt{t} S_{2}(\tau)\right)^{2 / p_{2}}}\right) 1_{\{\xi>0, Q\}}\right\} \\
& \leq \frac{1}{2} \mathbf{E}\left\{\exp \left(-c(\varepsilon) t \int_{2 \tau_{1}}^{1} \frac{d \tau}{\left(1+\sqrt{t} S_{2}(\tau)\right)^{2 / p_{2}}}\right)\right\} .
\end{aligned}
$$

On the other hand, in the case of $\left\{\xi>0, Q^{c}\right\}=\left\{(1+\sqrt{t} \xi)^{2 / p_{1}}>(1+\right.$ $\left.\left.\sqrt{t} S_{2}\left(2 \tau_{1}\right)\right)^{2 / p_{2}}\right\}$, in view of the monotonicity of $S_{2}(t)$, for the integral in $I V$, we have

$$
\int_{\tau_{1}}^{1} \frac{d \tau}{\max \left\{(1+\sqrt{t} \xi)^{2 / p_{1}},\left(1+\sqrt{t} S_{2}(\tau)\right)^{2 / p_{2}}\right\}} \geq \frac{\tau_{1}}{(1+\sqrt{t} \xi)^{2 / p_{1}}} .
$$

Plugging (3.18) into $I V$ yields

$$
\begin{aligned}
I V & \leq \mathbf{E}\left\{\exp \left(-\frac{c(\varepsilon) \tau_{1} t}{(1+\sqrt{t} \xi)^{2 / p_{1}}}\right) 1_{\left\{\xi>0, Q^{c}\right\}}\right\} \\
& \leq \mathbf{E}\left\{\exp \left(-\frac{c(\varepsilon) \tau_{1} t^{\left(p_{1}-1\right) / p_{1}}}{\left(t^{-1 / 2}+\xi\right)^{2 / p_{1}}}\right) 1_{\{\xi>0\}}\right\} .
\end{aligned}
$$

Using (3.15) and (3.17), we have

$$
I+I I I \leq \mathbf{E}\left\{\exp \left(-c(\varepsilon) t^{\left(p_{2}-1\right) / p_{2}} \int_{2 \tau_{1}}^{1} \frac{d \tau}{\left(t^{-1 / 2}+S_{2}(\tau)\right)^{2 / p_{2}}}\right)\right\} .
$$


Next we try to find the upper estimates of $I+I I I$ and $I V$, respectively. We split the expectation in (3.20) into two parts,

$$
\begin{aligned}
& \mathbf{E}\left\{\exp \left(-c(\varepsilon) t^{\left(p_{2}-1\right) / p_{2}} \int_{2 \tau_{1}}^{1} \frac{d \tau}{\left(t^{-1 / 2}+S_{2}(\tau)\right)^{2 / p_{2}}}\right)\right\} \\
\leq & \mathbf{E}\left\{\exp \left(-c(\varepsilon) t^{\left(p_{2}-1\right) / p_{2}} \int_{3 \tau_{1}}^{1} \frac{d \tau}{\left(t^{-1 / 2}+S_{2}(\tau)\right)^{2 / p_{2}}}\right) 1_{\left\{t^{-1 / 2} \leq \varepsilon S_{2}\left(3 \tau_{1}\right)\right\}}\right\} \\
& +\mathbf{E}\left\{\exp \left(-c(\varepsilon) t^{\left(p_{2}-1\right) / p_{2}} \int_{2 \tau_{1}}^{3 \tau_{1}} \frac{d \tau}{\left(t^{-1 / 2}+S_{2}(\tau)\right)^{2 / p_{2}}}\right) 1_{\left\{t^{-1 / 2}>\varepsilon S_{2}\left(3 \tau_{1}\right)\right\}}\right\} \\
\leq & \mathbf{E}\left\{\exp \left(-\frac{c(\varepsilon) t^{\left(p_{2}-1\right) / p_{2}}}{(1+\varepsilon)^{2 / p_{2}}} \int_{3 \tau_{1}}^{1} \frac{d \tau}{S_{2}^{2 / p_{2}}(\tau)}\right)\right\}+\exp \left(-\frac{c(\varepsilon) \tau_{1} t}{\left(1+\varepsilon^{-1}\right)^{2 / p_{2}}}\right) .
\end{aligned}
$$

At this stage, using Lemma 2.4 for the first term on the right-hand side of (3.21), we get that

$$
\begin{aligned}
& \inf _{\delta>0, \theta>0} \limsup _{t \rightarrow \infty} t^{-\frac{p_{2}-1}{p_{2}+1}} \log \mathbf{E}\left\{\exp \left(-(1-\delta) d(t) t^{\frac{p_{2}-1}{p_{2}}} \int_{\theta}^{1} \frac{d \tau}{S_{2}^{2 / p_{2}}(\tau)}\right)\right\} \\
& \leq-\left(p_{2}+1\right)\left(d(t) / p_{2}\right)^{p_{2} /\left(p_{2}+1\right)} B_{0, p_{2}}^{1 /\left(p_{2}+1\right)} .
\end{aligned}
$$

Next, dealing with $I V$. For the sake of brevity, write $U_{1}=\left\{0<\xi \leq t^{-1 / 2}\right\}$,

$$
\begin{gathered}
U_{2}=\left\{t^{-1 / 2}<\xi \leq 2^{\frac{p_{1}-2}{2\left(p_{1}+1\right)}}\left(c(\varepsilon) \tau_{1} p_{1}^{-1}\right)^{\frac{p_{1}}{2\left(p_{1}+1\right)}} t^{\frac{p_{1}-1}{2\left(p_{1}+1\right)}}\right\}, \\
U_{3}=\left\{2^{\frac{p_{1}-2}{2\left(p_{1}+1\right)}}\left(c(\varepsilon) \tau_{1} p_{1}^{-1}\right)^{\frac{p_{1}}{2\left(p_{1}+1\right)}} t^{\frac{p_{1}-1}{2\left(p_{1}+1\right)}}<\xi<\infty\right\} .
\end{gathered}
$$

We split the expectation on the right side of (3.19) into three parts. Writing them as $V, V I$ and $V I I$. Next, we deal with the three expectations, respectively. Firstly,

$$
\begin{aligned}
V & =\mathbf{E}\left\{\exp \left(-\frac{c(\varepsilon) \tau_{1} t^{\left(p_{1}-1\right) / p_{1}}}{\left(t^{-1 / 2}+\xi\right)^{2 / p_{1}}}\right) 1_{\left\{U_{1}\right\}}\right\} \\
& \leq(2 \pi)^{-1 / 2} t^{-1 / 2} \exp \left(-c(\varepsilon) \tau_{1} 2^{-2 / p_{1}} t\right) .
\end{aligned}
$$

Secondly,

$$
\begin{aligned}
V I & =\mathbf{E}\left\{\exp \left(-\frac{c(\varepsilon) \tau_{1} t^{\left(p_{1}-1\right) / p_{1}}}{\left(t^{-1 / 2}+\xi\right)^{2 / p_{1}}}\right) 1_{\left\{U_{2}\right\}}\right\} \\
& \leq \frac{1}{\sqrt{2 \pi}} \int_{t^{-1 / 2}}^{k t^{\frac{p_{1}-1}{2\left(p_{1}+1\right)}}} \exp \left\{-\left(\frac{c(\varepsilon) \tau_{1} t^{\left(p_{1}-1\right) / p_{1}}}{2^{2 / p_{1}} u^{2 / p_{1}}}+\frac{u^{2}}{2}\right)\right\} d u \\
& \leq \frac{k}{\sqrt{2 \pi}} t^{\frac{p_{1}-1}{2\left(p_{1}+1\right)}} \exp \left\{-\inf _{t^{-\frac{1}{2}}<u \leq k t^{\frac{p_{1}-1}{2\left(p_{1}+1\right)}}}\left(\frac{c(\varepsilon) \tau_{1} t^{\frac{p_{1}-1}{p_{1}}}}{2^{2 / p_{1}} u^{2 / p_{1}}}+\frac{u^{2}}{2}\right)\right\} \\
& =(2 \pi)^{-1 / 2} k t^{\frac{p_{1}-1}{2\left(p_{1}+1\right)}} \exp \left\{-2^{-\frac{3}{p_{1}+1}}\left(c(\varepsilon) \tau_{1} p_{1}^{-1}\right)^{\frac{p_{1}}{p_{1}+1}}\left(p_{1}+1\right) t^{\frac{p_{1}-1}{p_{1}+1}}\right\},
\end{aligned}
$$


where $k=2^{\frac{p_{1}-2}{2\left(p_{1}+1\right)}}\left(c(\varepsilon) \tau_{1} p_{1}^{-1}\right)^{\frac{p_{1}}{2\left(p_{1}+1\right)}}$. Note that the inf in (3.24) is attained at $u=k t^{\left(p_{1}-1\right) / 2\left(p_{1}+1\right)}$. Finally,

$$
\begin{aligned}
V I I & =\mathbf{E}\left\{\exp \left(-\frac{c(\varepsilon) \tau_{1} t^{\left(p_{1}-1\right) / p_{1}}}{\left(t^{-1 / 2}+\xi\right)^{2 / p_{1}}}\right) 1_{\left\{U_{3}\right\}}\right\} \\
& \leq \frac{1}{\sqrt{2 \pi}} \int_{k t^{\frac{p_{1}-1}{2\left(p_{1}+1\right)}}}^{\infty} \exp \left\{-2^{-1} u^{2}\right\} d u \\
& \leq(2 \pi)^{-1 / 2} k^{-1} t^{-\frac{p_{1}-1}{2\left(p_{1}+1\right)}} \exp \left\{-2^{-1} k^{2} t^{\frac{p_{1}-1}{p_{1}+1}}\right\} .
\end{aligned}
$$

The second inequality in (3.25) can be easily obtained from the result of Mill's ratio (see [6]). Comparing (3.22)-(3.25), it is easy to see that (3.22) is the dominant term, then using all above inequalities, we have

$$
\begin{aligned}
& \limsup _{t \rightarrow \infty} t^{-\left(p_{2}-1\right) /\left(p_{2}+1\right)} \log P\left(\|B(s)\| \leq \max _{j=1,2}\left\{\left(1+W_{j}(s)\right)^{1 / p_{j}}\right\}, 0 \leq s \leq t\right) \\
\leq & \inf _{\delta>0, \theta>0} \limsup _{t \rightarrow \infty} t^{-\frac{p_{2}-1}{p_{2}+1}} \log \mathbf{E}\left\{\exp \left(-(1-\delta) d(t) t^{\frac{p_{2}-1}{p_{2}}} \int_{\theta}^{1} \frac{d \tau}{S_{2}^{2 / p_{2}}(\tau)}\right)\right\} \\
\leq & -\left(p_{2}+1\right)\left(d(t) / p_{2}\right)^{p_{2} /\left(p_{2}+1\right)} B_{0, p_{2}}^{1 /\left(p_{2}+1\right)} .
\end{aligned}
$$

\section{Lower estimates}

It is easy to obtain the lower estimate of (1.12). Note that

$$
\begin{aligned}
& P\left(\|B(s)\| \leq \max _{j=1,2}\left\{\left(1+W_{j}(s)\right)^{1 / p_{j}}\right\}, 0 \leq s \leq t\right) \\
\geq & P\left(\bigcup_{j=1,2}\left\{\|B(s)\|^{p_{j}} \leq 1+W_{j}(s), 0 \leq s \leq t\right\}\right) \\
\geq & \max _{j=1,2}\left\{P\left(\|B(s)\|^{p_{j}} \leq 1+W_{j}(s), 0 \leq s \leq t\right)\right\} .
\end{aligned}
$$

On the right-hand side of (4.1), we use Lemma 2.5 to find the lower bounds of two probabilities, and compare them to get the lower estimates in (1.12) as follows:

$$
\begin{aligned}
& \liminf _{t \rightarrow \infty} t^{-\left(p_{2}-1\right) /\left(p_{2}+1\right)}(d(t))^{-2 p_{2} /\left(p_{2}+1\right)} \log P\left(\|B(s)\| \leq \max _{j=1,2}\left\{\left(1+W_{j}(s)\right)^{1 / p_{j}}\right\}, 0 \leq s \leq t\right) \\
\geq & -\left(p_{2}+1\right)\left(\frac{c_{2}^{3 p_{2}} \pi}{8\left(p_{2}-1\right)^{p_{2}-1}} \cdot \frac{\Gamma^{2}\left(\left(p_{2}-1\right) / 2\right)}{\Gamma^{2}\left(p_{2} / 2\right)}\right)^{\frac{1}{p_{2}+1}} .
\end{aligned}
$$

Next, we give the proof of the lower bound in (1.14). Our argument is a modification of the one from [5]. Take a function $h \in A_{0, p_{1}}^{\uparrow}$ solving the variational problem

$$
B_{0, p_{1}}:=\frac{1}{2} \inf _{f \in A_{0, p_{1}}^{\uparrow}} \int_{0}^{1} \dot{f}^{2}(s) d s
$$


where $A_{0, p_{1}}^{\uparrow}$ is the set of all non-decreasing functions on the set $A_{0, p_{1}}$ defined by $A_{0, p_{1}}:=\left\{f:[0,1] \rightarrow R_{+}, f(0)=0, f\right.$ absolutely continuous, $\left.\int_{0}^{1} f^{-2 / p_{1}}(s) d s \leq 1\right\}$.

That is, let $h \in A_{0, p_{1}}^{\uparrow}$ be such that

$$
\frac{1}{2} \int_{0}^{1} \dot{h}^{2}(s) d s=B_{0, p_{1}} \quad \text { and } \quad \int_{0}^{1} h^{-2 / p_{1}}(s) d s=1 .
$$

From (4.4), it is easy to verify that $h(x)=o\left(x^{1 / 2}\right)$, and $x^{p_{1} / 2}=o(h(x))$ for $x \rightarrow 0^{+}$. Since $p_{1}>p_{2}>1$, there exists $2>r>1$, such that $p_{1}>p_{2}>r>1$. Consider an approximation $h_{1}$ of $h$ such that for any $\varepsilon>0$,

$$
h_{1}(s)=h(\varepsilon) \varepsilon^{-r / 2} s^{r / 2}, s \in[0, \varepsilon] \quad \text { and } \quad h_{1}(s)=h(s), s \in[\varepsilon, 1] .
$$

It is easy to see that $h_{1}$ is nondecreasing and absolutely continuous function. Note that for $\varepsilon \rightarrow 0^{+}$,

$$
\frac{1}{2} \int_{0}^{\varepsilon} \dot{h}_{1}^{2}(s) d s=\frac{r^{2} h^{2}(\varepsilon)}{8(r-1) \varepsilon} \rightarrow 0 \quad \text { and } \quad \int_{0}^{\varepsilon} h_{1}^{-2 / p_{1}}(s) d s=\frac{p_{1}}{p_{1}-r} \frac{\varepsilon}{h^{2 / p_{1}}(\varepsilon)} \rightarrow 0 .
$$

Thus, for any $\delta>0$ and sufficiently small $\varepsilon$,

$$
\frac{1}{2} \int_{0}^{1} \dot{h}_{1}^{2}(s) d s \leq(1+\delta) B_{0, p_{1}} \quad \text { and } \quad \int_{0}^{1} h_{1}^{-2 / p_{1}}(s) d s \leq 1+\delta .
$$

Using Li's profile function method from [4], we have

$$
\begin{aligned}
& P\left(\|B(s)\| \leq \min _{j=1,2}\left\{\left(1+W_{j}(s)\right)^{1 / p_{j}}\right\}, 0 \leq s \leq t\right) \\
= & P\left(\bigcap_{j=1,2}\left\{\|B(s)\|^{p_{j}} \leq 1+W_{j}(s), 0 \leq s \leq t\right\}\right) \\
\geq & P\left(\left\{\|B(s)\|^{p_{1}} \leq \rho t^{p_{1} /\left(p_{1}+1\right)} h_{1}(s / t) \leq 1+W_{1}(s), 0 \leq s \leq t\right\},\right. \\
& \left.\left\{\|B(s)\|^{p_{2}} \leq \rho^{p_{2} / p_{1}} t^{p_{2} /\left(p_{2}+1\right)} h_{1}(s / t) \leq 1+W_{2}(s), 0 \leq s \leq t\right\}\right),
\end{aligned}
$$

where the optimal value of the additional parameter $\rho>0$ is yet to be chosen. By the independence between the processes $W_{j}, j=1,2$ and $B$, we have

$$
\begin{aligned}
& P\left(\|B(s)\| \leq \min _{j=1,2}\left\{\left(1+W_{j}(s)\right)^{1 / p_{j}}\right\}, 0 \leq s \leq t\right) \\
\geq & P\left(\|B(s)\| \leq \rho^{1 / p_{1}} \min \left\{t^{1 /\left(p_{1}+1\right)} h_{1}^{1 / p_{1}}(s / t), t^{1 /\left(p_{2}+1\right)} h_{1}^{1 / p_{2}}(s / t)\right\}, 0 \leq s \leq t\right) \\
& \times P\left(\rho t^{p_{1} /\left(p_{1}+1\right)} h_{1}(s / t) \leq 1+W_{1}(s), 0 \leq s \leq t\right) \\
& \times P\left(\rho^{p_{2} / p_{1}} t^{p_{2} /\left(p_{2}+1\right)} h_{1}(s / t) \leq 1+W_{2}(s), 0 \leq s \leq t\right) .
\end{aligned}
$$


For the first probability on the right-hand side of (4.7), by the scaling property of $B$, we have

$$
\begin{aligned}
& P\left(\|B(s)\| \leq \rho^{\frac{1}{p_{1}}} \min \left\{t^{\frac{1}{p_{1}+1}} h_{1}^{\frac{1}{p_{1}}}(s / t), t^{\frac{1}{p_{2}+1}} h_{1}^{\frac{1}{p_{2}}}(s / t)\right\}, 0 \leq s \leq t\right) \\
= & P\left(\|B(s)\| \leq \rho^{\frac{1}{p_{1}}} \min \left\{t^{\frac{1-p_{1}}{2\left(p_{1}+1\right)}} h_{1}^{\frac{1}{p_{1}}}(s), t^{\frac{1-p_{2}}{2\left(p_{2}+1\right)}} h_{1}^{\frac{1}{p_{2}}}(s)\right\}, 0 \leq s \leq 1\right) \\
= & P\left(\|B(s)\| \leq t^{-\frac{p_{1}-1}{2\left(p_{1}+1\right)}} \rho^{\frac{1}{p_{1}}} \min \left\{h_{1}^{\frac{1}{p_{1}}}(s), t^{\frac{p_{1}-1}{2\left(p_{1}+1\right)}-\frac{p_{2}-1}{2\left(p_{2}+1\right)}} h_{1}^{\frac{1}{p_{2}}}(s)\right\}, 0 \leq s \leq 1\right) .
\end{aligned}
$$

Choosing

$$
\nu>\max \left\{(h(\varepsilon))^{-\frac{\left(p_{1}+1\right)\left(p_{2}+1\right)}{p_{1} p_{2}}},\left(\frac{\varepsilon r\left(p_{1}-p_{2}\right)}{h^{2 / r}(\varepsilon)\left(p_{1}-r\right)\left(p_{2}-r\right) \delta}\right)^{\frac{r\left(p_{1}+1\right)\left(p_{2}+1\right)}{2 p_{2}\left(p_{1}-r\right)}}\right\},
$$

we see that

$$
h(\varepsilon)>\nu^{-p_{1} p_{2} /\left(p_{1}+1\right)\left(p_{2}+1\right)}, \quad \frac{\varepsilon r\left(p_{1}-p_{2}\right)}{h^{2 / r}(\varepsilon)\left(p_{1}-r\right)\left(p_{2}-r\right)} \nu^{-\frac{2 p_{2}\left(p_{1}-r\right)}{r\left(p_{1}+1\right)\left(p_{2}+1\right)}}<\delta .
$$

Plugging $\nu$ into the last probability in (4.8) and using the inequality (2.3) in Propsition 2.1, we have

$$
\begin{aligned}
& \liminf _{t \rightarrow \infty} t^{-\frac{p_{1}-1}{p_{1}+1}} \log P\left(\|B(s)\| \leq t^{-\frac{p_{1}-1}{2\left(p_{1}+1\right)}} \rho^{\frac{1}{p_{1}}} \min \left\{h_{1}^{\frac{1}{p_{1}}}(s), t^{\frac{p_{1}-1}{2\left(p_{1}+1\right)}-\frac{p_{2}-1}{2\left(p_{2}+1\right)}} h_{1}^{\frac{1}{p_{2}}}(s)\right\}, 0 \leq s \leq 1\right) \\
\geq & \liminf _{t \rightarrow \infty} t^{-\frac{p_{1}-1}{p_{1}+1}} \log P\left(\|B(s)\| \leq t^{-\frac{p_{1}-1}{2\left(p_{1}+1\right)}} \rho^{\frac{1}{p_{1}}} \min \left\{h_{1}^{\frac{1}{p_{1}}}(s), \nu^{\frac{p_{1}-1}{2\left(p_{1}+1\right)}-\frac{p_{2}-1}{2\left(p_{2}+1\right)}} h_{1}^{\frac{1}{p_{2}}}(s)\right\}, 0 \leq s \leq 1\right) \\
\geq & -\frac{(1+\delta) c_{2}^{3} d^{2}(t)}{\rho^{2 / p_{1}}} \int_{0}^{1} \frac{d s}{\min \left\{h_{1}^{2 / p_{1}}(s), \nu^{\left(p_{1}-1\right) /\left(p_{1}+1\right)-\left(p_{2}-1\right) /\left(p_{2}+1\right)} h_{1}^{2 / p_{2}}(s)\right\}} .
\end{aligned}
$$
If

Next, we try to find the minimum between $h_{1}^{2 / p_{1}}(s)$ and $\nu^{\left(p_{1}-1\right) /\left(p_{1}+1\right)-\left(p_{2}-1\right) /\left(p_{2}+1\right)} h_{1}^{2 / p_{2}}(s)$.

$$
h_{1}^{2 / p_{1}}(s) \leq \nu^{\left(p_{1}-1\right) /\left(p_{1}+1\right)-\left(p_{2}-1\right) /\left(p_{2}+1\right)} h_{1}^{2 / p_{2}}(s),
$$

then we have

$$
h_{1}(s) \geq \nu^{-p_{1} p_{2} /\left(p_{1}+1\right)\left(p_{2}+1\right)} .
$$

From the inequalities (4.9), we have

$$
h_{1}(\varepsilon)=h(\varepsilon)>\nu^{-p_{1} p_{2} /\left(p_{1}+1\right)\left(p_{2}+1\right)} .
$$

Recall that

$$
h_{1}(s)=h(\varepsilon) \varepsilon^{-r / 2} s^{r / 2}, s \in[0, \varepsilon] \quad \text { and } \quad h_{1}(s)=h(s), s \in[\varepsilon, 1] .
$$

Since $h_{1}(s)$ is non-decreasing on $[0,1]$, it is easy to see that for

$$
s \in V=\left[\varepsilon h(\varepsilon)^{-2 / r} \nu^{-2 p_{1} p_{2} / r\left(p_{1}+1\right)\left(p_{2}+1\right)}, 1\right],
$$

we have

$$
h_{1}^{2 / p_{1}}(s) \leq \nu^{\left(p_{1}-1\right) /\left(p_{1}+1\right)-\left(p_{2}-1\right) /\left(p_{2}+1\right)} h_{1}^{2 / p_{2}}(s) .
$$


Note that $1 \geq h(\varepsilon)^{-1} \nu^{-p_{1} p_{2} /\left(p_{1}+1\right)\left(p_{2}+1\right)}$ forces $s<\varepsilon$ for $s \in V^{c}$. This is used in formula (4.11). Then splitting the integral in (4.10) into two parts, we get

$$
\begin{aligned}
& \int_{0}^{1} \frac{d s}{\min \left\{h_{1}^{2 / p_{1}}(s), \nu^{\left(p_{1}-1\right) /\left(p_{1}+1\right)-\left(p_{2}-1\right) /\left(p_{2}+1\right)} h_{1}^{2 / p_{2}}(s)\right\}} \\
= & \int_{V^{c}} \frac{d s}{h_{1}^{2 / p_{2}}(s)} \nu^{\frac{p_{2}-1}{p_{2}+1}-\frac{p_{1}-1}{p_{1}+1}}+\int_{V} \frac{d s}{h_{1}^{2 / p_{1}}(s)} .
\end{aligned}
$$

It is easy to verify that

$$
\begin{gathered}
\int_{V^{c}} \frac{d s}{h_{1}^{2 / p_{1}}(s)}=\int_{V^{c}} \frac{d s}{\left(h(\varepsilon) \varepsilon^{-r / 2} s^{r / 2}\right)^{2 / p_{1}}}=\frac{\varepsilon p_{1}}{h^{2 / r}(\varepsilon)\left(p_{1}-r\right)} \nu^{-\frac{2 p_{2}\left(p_{1}-r\right)}{r\left(p_{1}+1\right)\left(p_{2}+1\right)}} \\
\int_{V^{c}} \frac{d s}{h_{1}^{2 / p_{2}}(s)} \nu^{\frac{p_{2}-1}{p_{2}+1}-\frac{p_{1}-1}{p_{1}+1}}=\frac{\varepsilon p_{2}}{h^{2 / r}(\varepsilon)\left(p_{2}-r\right)} \nu^{-\frac{2 p_{2}\left(p_{1}-r\right)}{r\left(p_{1}+1\right)\left(p_{2}+1\right)}}
\end{gathered}
$$

Using (4.8)-(4.13) and (4.5), we have

$$
\begin{aligned}
& \liminf _{t \rightarrow \infty} t^{-\frac{p_{1}-1}{p_{1}+1}} \log P\left(\|B(s)\| \leq \rho^{\frac{1}{p_{1}}} \min \left\{t^{\frac{1}{p_{1}+1}} h_{1}^{\frac{1}{p_{1}}}(s / t), t^{\frac{1}{p_{2}+1}} h_{1}^{\frac{1}{p_{2}}}(s / t)\right\}, 0 \leq s \leq t\right) \\
\geq & -\frac{(1+\delta) c_{2}^{3} d^{2}(t)}{\rho^{2 / p_{1}}}\left(\int_{0}^{1} \frac{d s}{h_{1}^{2 / p_{1}}(s)}+\int_{V^{c}} \frac{d s}{h_{1}^{2 / p_{2}}(s)} \nu^{\frac{p_{2}-1}{p_{2}+1}-\frac{p_{1}-1}{p_{1}+1}}-\int_{V^{c}} \frac{d s}{h_{1}^{2 / p_{1}}(s)}\right) \\
= & -\frac{(1+\delta) c_{2}^{3} d^{2}(t)}{\rho^{2 / p_{1}}}\left(\int_{0}^{1} \frac{d s}{h_{1}^{2 / p_{1}}(s)}+\frac{\varepsilon r\left(p_{1}-p_{2}\right)}{h^{2 / r}(\varepsilon)\left(p_{1}-r\right)\left(p_{2}-r\right)} \nu^{-\frac{2 p_{2}\left(p_{1}-r\right)}{r\left(p_{1}+1\right)\left(p_{2}+1\right)}}\right) \\
\geq & -(1+2 \delta)(1+\delta) \frac{c_{2}^{3} d^{2}(t)}{2 \rho^{2 / p_{1}}} .
\end{aligned}
$$

For the second and third probabilities on the right-hand side of (4.7), let $q>1$. By taking $p=q$ in Lemma 2.3, we can use both (2.13) and (4.5) to get

$$
\begin{aligned}
& \liminf _{t \rightarrow \infty} t^{-\frac{p_{1}-1}{p_{1}+1}} \log P\left(\rho t^{p_{1} /\left(p_{1}+1\right)} h_{1}(s / t) \leq 1+W_{1}(s), 0 \leq s \leq t\right)(4.15 \\
\geq & -\frac{q \rho^{2}}{2} \int_{0}^{1} \dot{h}_{1}^{2}(s) d s \\
\geq & -q \rho^{2}(1+\delta) B_{0, p_{1}}
\end{aligned}
$$

and

$$
\begin{aligned}
& \liminf _{t \rightarrow \infty} t^{-\frac{p_{2}-1}{p_{2}+1}} \log P\left(\rho^{p_{2} / p_{1}} t^{p_{2} /\left(p_{2}+1\right)} h_{1}(s / t) \leq 1+W_{2}(s), 0 \leq s \leq t\right) \\
\geq & -\frac{q \rho^{2 p_{2} / p_{1}}}{2} \int_{0}^{1} \dot{h}_{1}^{2}(s) d s \\
\geq & -q \rho^{2 p_{2} / p_{1}}(1+\delta) B_{0, p_{1}} .
\end{aligned}
$$


Comparing (4.14) and (4.15) with (4.16), it is easy to see that (4.14) and (4.15) are the dominant terms. Using (4.7), we have

$$
\begin{aligned}
& \liminf _{t \rightarrow \infty} t^{-\left(p_{1}-1\right) /\left(p_{1}+1\right)} \log P\left(\|B(s)\| \leq \min _{i=1,2}\left\{\left(1+W_{i}(s)\right)^{1 / p_{i}}\right\}, 0 \leq s \leq t\right) \\
\geq & -(1+2 \delta)(1+\delta) \frac{c_{2}^{3} d^{2}(t)}{\rho^{2 / p_{1}}}-(1+\delta) q \rho^{2} B_{0, p_{1}} .
\end{aligned}
$$

By letting $q \rightarrow 1$ and $\delta \rightarrow 0$, then maximizing the term on the right hand side via the choice

$$
\rho:=\left(c_{2}^{3} d^{2}(t) / p_{1} B_{0, p_{1}}\right)^{p_{1} / 2\left(p_{1}+1\right)},
$$

we obtain

$$
\begin{aligned}
& \liminf _{t \rightarrow \infty} t^{-\left(p_{1}-1\right) /\left(p_{1}+1\right)} \log P\left(\|B(s)\| \leq \min _{i=1,2}\left\{\left(1+W_{i}(s)\right)^{1 / p_{i}}\right\}, 0 \leq s \leq t\right) \\
\geq & -\left(p_{1}+1\right)\left(c_{2}^{3} d^{2}(t) / p_{1}\right)^{p_{1} /\left(p_{1}+1\right)} B_{0, p_{1}}^{1 /\left(p_{1}+1\right)} .
\end{aligned}
$$

This completes the proof of Theorem 1.1.

\section{Conclusion}

This paper proves that the estimation of the upper and lower bounds during the first exit time of the Brownian motion is meaningful for the species ecological chain problem in the biological world.

\section{Ethics approval and consent to participate}

Not applicable

\section{Consent for publication}

Not applicable

\section{Funding}

Not applicable

\section{Availability of data and material}

This article is intended to prove the theoretical study of species in the ecosphere, without data.

\section{Competing interests}

The authors declare that they have no competing interests. 


\section{Authors' contributions}

The first author(Chao Liu) conceived the innovation of this paper and provided relevant lemmas to prove the theory. The second author(Wenbin Che) and the third author(Jingjun Zhang) gave detailed proof of the main conclusion of the paper.

Acknowledgements We thank the referees for careful reading of our manuscrip$\mathrm{t}$ and for helpful and valuable comments and suggestions.

\section{Appendix}

A.1. Explanation of mathematical symbols and formulas

\begin{tabular}{|c|c|}
\hline Abbreviations & Full name \\
\hline $\mathrm{B}\left({ }^{*}\right)$ & Brownian motion \\
\hline $\mathrm{P}(*)$ & Probability function \\
\hline$\|*\|$ & Euclidean norm \\
\hline $\mathrm{E}^{*}$ & Expectation \\
\hline$* \lim$ & limit \\
\hline liminf & limit inferior \\
\hline limsup & limit superior \\
\hline${ }^{*} \max$ & maximum \\
\hline${ }^{*} \min$ & minimum \\
\hline${ }^{*} \inf$ & infimum \\
\hline$*$ sup & supremum \\
\hline
\end{tabular}

\section{References}

[1] Berthet, Ph. and Shi, Z.: Small ball estimates for Brownian motion under a weighted sup-norm. Studia Sci. Math. Hungar. 36, 275-289 (2000)

[2] Ciesielski, Z. and Taylor, S. J.: First passage time and sojourn times for Brownian motion in space and the exact Hausdorff measure of the sample path. Trans. Amer. Math. Soc. 103(3), 434-450 (1962)

[3] Gordon, Y.: Some inequalities for Gaussian processes and applications. Israel J. Math. 50(4), 265-289 (1985)

[4] Li, W.V.: The first exit time of a Brownian motion from an unbounder convex domain. Ann. Probab. 31(2), 1078-1096 (2003)

[5] Lifshits, M.A. and Shi, Z.: The first exit time of Brownian motion from a parabolic domain. Bernoulli. 8(6), 745-765 (2002)

[6] D. Lu, L. Song, The first exit time of a Brownian motion from the minimum and maximum parabolic domains, J. Theor. Probab., Publish on line. (2011). 
[7] Novikov, A.A.: On estimates and asymptotic behavior for non-exit probabilities of a Wiener process to a moving boundary. Mat. Sb. 38(4), 495-505 (1984)

[8] Q.M. Shao, A note on small ball probability of Gaussian process with stationary increments. J. Theor. Probab. 6, 595-602.(1993).

[9] Q.M. Shao, D.Y. Wang, Small ball probabilities of Gaussian fields. Probab. Theory Rel. Fields, 12,511-517.(1995).

[10] L. Song, Upper and lower bounds of the first exit time of Brownian motion from a parabolic domain with variable dimension. J. Theor. Probab.

[11] Rockafellar, R.T.: Convex Analysis. Princeton Univ Press, Princeton (1970) 